\title{
The Treatment of Acute Myelogenous Leukemia
}

\author{
R. Bassan, A.Z.S. Rohatiner, W. Gregory, J. Amess, R. Biruls, M. J. Barnett, and T. A. \\ Lister
}

\section{A. Introduction}

It has recently been suggested that it is possible to cure at least $25 \%$ of younger adults who have acute myelogenous leukaemia [1]. The results achieved at St. Bartholomew's Hospital, London, demonstrate this and may be used to illustrate the still outstanding problems.

\section{B. Patients and Methods}

One hundred and eighty-five consecutive, previously untreated patients, aged 15-59 years, commenced short-term chemotherapy between 1978 and 1986, some as part of three sequential open studies and some in a randomised clinical trial. The majority (106), who received BX therapy comprising an intended six cycles of adriamycin $(25 \mathrm{mg}$ / $\mathrm{m}^{2}$ days 1,2 , and 3 ), cytosine arabinoside by i.v. bolus $\left(100 \mathrm{mg} / \mathrm{m}^{2}\right.$ twice daily) and 6 thioguanine orally ( $200 \mathrm{mg}$ daily), each of the last two for 7 days, form the basis of this analysis. The intercycle (day 1-day 1) time was proposed to be 21 days but ranged from 18 to 66 days, depending on the clinical state of the patient and the morphological appearance of the bone marrow.

Patients were cared for in an open ward. Bowel decontamination was attempted with non-absorbable antibiotics; fever was assumed to be due to bacterial infection and was treated with appropriate antibiotic com-

ICRF Department of Medical Oncology, St. Bartholomew's Hospital, London, England binations, and prophylactic platelet transfusions were given to maintain the platelet count above $20 \times 10^{9} / 1$.

\section{Results}

Complete remission was achieved in 64 of 106 patients; the reasons for failure are shown in Table 1. Of these 64, 34 patients continue in unmaintained complete remission with a median follow-up of 1 year (four proceeded to allogenic or syngeneic bone marrow transplantation, and have been "censured" since that time). Thirtyseven others are alive, five in second or subsequent complete remission. All deaths were attributable to leukaemia (at presentation or relapse) or its therapy (Fig. 1). Multivariate analysis demonstrated that the only factor to correlate either with prolonged freedom from leukaemia or with survival was the interval between cycles 1 and 2 . The other factors considered were:

- Age

- FAB classification

- Blast count

- Serum albumin

Table 1. Responses to BX therapy among 106 patients

\begin{tabular}{lc}
\hline Complete remission & $64(60 \%)$ \\
Resistant leukaemia & 11 \\
Supportive-care failure & 30 \\
Other cause & 1 \\
Total & 106 \\
\hline
\end{tabular}




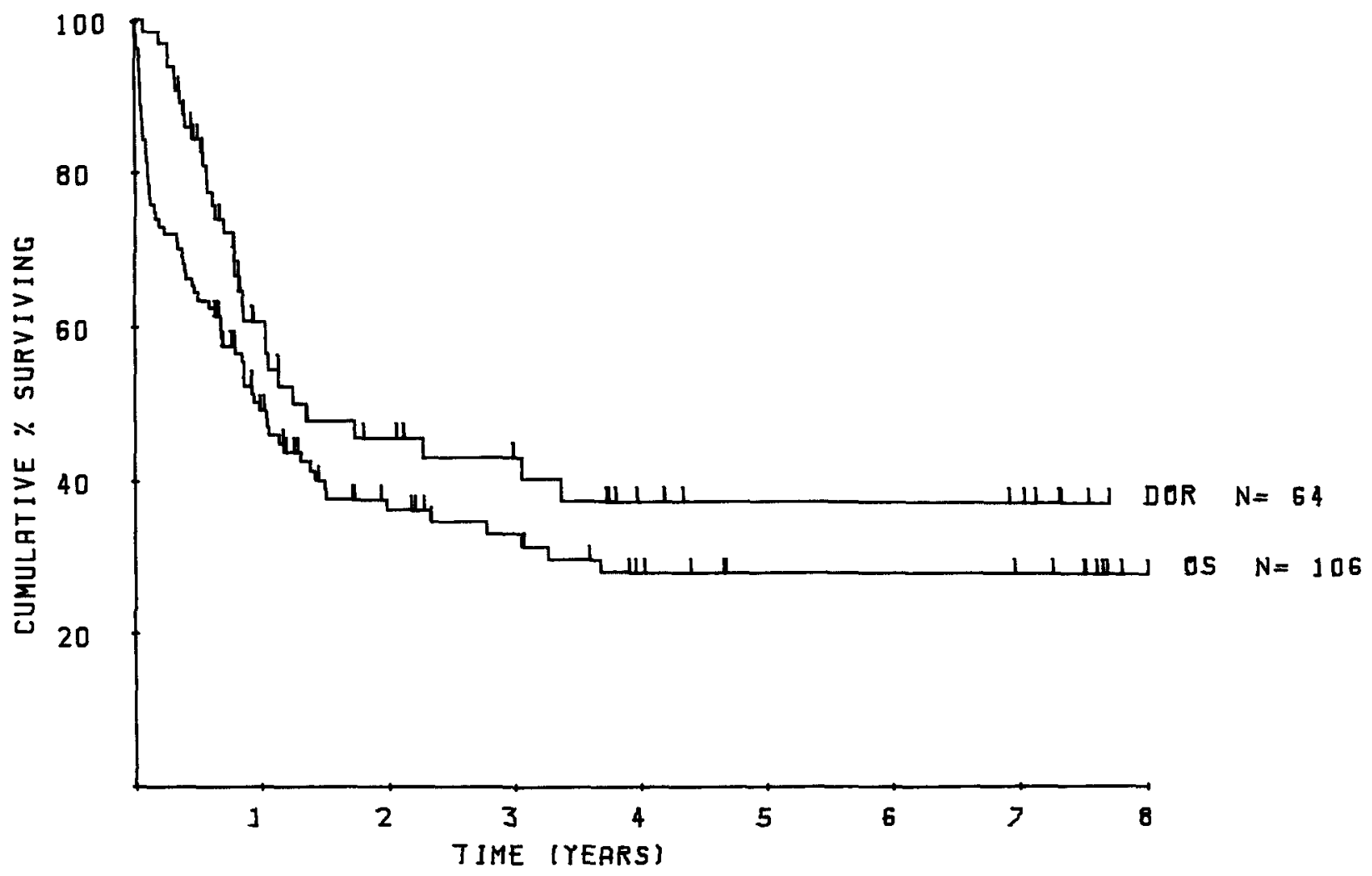

Fig. 1. Overall survival $(O S)$ and duration of remission $(D O R)$ in patients aged 15-59 years

- Hepatosplenomegaly

- Total no. of treatment cycles

- Cycles of treatment to achieve CR

- Cycles of consolidation after CR

There was no correlation between the number of cycles of therapy received (beyond a minimum of three) and duration of remission.

\section{Discussion}

These results clearly demonstrate that cure is possible for at least $25 \%$ of younger patients with acute myelogenous leukaemia. They identify failure to eradicate leukaemia as by far the greatest problem, 11 patients having died of resistant disease and 30 having had a recurrence within 3 years. Previous results from St. Bartholomew's Hospital suggest that the likelihood of many recurrences after the 3-year point is small, although it does exist. It may be inferred that further attention must be given to very early treatment if the results are to be improved, and that prolonging therapy is unlikely to have a major effect. It may even be possible to reduce the duration of treatment and obtain equally good results, provided the intensity is maintained. Whether or not improvements will be achieved by increasing the number of drugs, altering the scheduling, or incorporating either allogeneic or autologous transplantation remains to be demonstrated.

It is probable, regardless of which approach is investigated, that prospective analyses of patient variables, particularly in cytogenetics, may identify those patients for whom the current strategy already carries a high probability of success, those for whom an alternative must be found if cure is to be achieved, and those who, no matter what the nature of the bone marrow defect, cannot tolerate the treatment and for whom it should not be prescribed.

\section{Reference}

1. Weinstein HJ, Mayer RJ, Rosenthal DS, Coral FS, Camitta BM, Gelber RD (1983) Chemotherapy for acute myelogenous leukemia in children and adults: VAPA update. Blood 62:315-319 